\title{
Authors' reply: Comment on: Long-term effects of bariatric surgery on cardiovascular risk factors in Singapore
}

Singapore Med J 2021; 62(9): 503 https://doi.org/10.11622/smedj.2021149

Dear Sir,

We appreciate and thank the writer for sharing his comments in response to our article. ${ }^{(1,2)}$

Although data on hypertension was not collected during our review, we have separately published mid-term hypertension outcomes of our patients following laparoscopic sleeve gastrectomy, wherein we reported that hypertension improves with weight loss in the mid term and is related to the degree of excess weight loss (EWL). Patients who achieved more than 35\% EWL had a $42.5 \%$ remission rate, compared with a remission rate of $6.8 \%$ among patients with less than $35 \%$ EWL. ${ }^{(3)}$ With regard to long-term outcomes, a systematic review in 2018 reported that the preoperative prevalence of hypertension was $36.5 \%$ (range $6.7 \%-91 \%$ ), which fell to $14.8 \%$ (range $0 \%-33 \%$ ) at the five-year follow-up. Hypertension resolved in $62.2 \%$ (range $0 \%-100 \%$ ) of patients and improved in $35.7 \%$ (range $13.3 \%-76.9 \%$ ) of patients at a mean of 5.35 years of follow-up. ${ }^{(4)}$

With regard to the difference between sleeve gastrectomy and Roux-en-Y gastric bypass in terms of lipid levels at the one-year and five-year follow-ups, the number of patients in our study was too small to perform such an analysis. A recent meta-analysis showed no difference in remission of high triglyceride levels and low high-density lipoprotein levels between the two procedures at five years; however, remission of high low-density lipoprotein levels and overall dyslipidaemia was superior in the group of patients who underwent Roux-en-Y gastric bypass. ${ }^{(5)}$

We hope that this explanation adequately addresses the issues raised in the letter.

Yours sincerely,

Vinay B $\underline{\text { Panday }}^{1}$, Asim $\underline{\text { Shabbir }}^{2}$, Ivandito $\underline{K u n t j o r}^{3}$, Eric Yin Hao $\underline{K h o O}^{1}$, Jimmy Bok Yan $\underline{\mathrm{So}}^{2,4}$, Kian Keong $\underline{\text { Poh }}^{3}$

${ }^{1}$ Department of Medicine, National University Health System, ${ }^{2}$ Department of Surgery, National University Hospital, ${ }^{3}$ Department of Cardiology, National University Heart Centre, National University Health System, ${ }^{4}$ NUS Yong Loo Lin School of Medicine, National University of Singapore, Singapore. vinaypanday36@gmail.com

\section{References}

1. Agrawal H. Comment on: Long-term effects of bariatric surgery on cardiovascular risk factors in Singapore. Singapore Med J 2021; 62:503.

2. Panday VB, Shabbir A, Kuntjoro I, et al. Long-term effects of bariatric surgery on cardiovascular risk factors in Singapore. Singapore Med J 2021; 62:472.

3. Chew CAZ, Tan IJW, Ng HJH, et al. Early weight loss after laparoscopic sleeve gastrectomy predicts midterm weight loss in morbidly obese Asians. Surg Obes Relat Dis 2017; 13:1966-72.

4. Graham C, Switzer N, Reso A, et al. Sleeve gastrectomy and hypertension: a systematic review of long-term outcomes. Surg Endosc 2019; 33:3001-7.

5. Gu L, Huang X, Li S, et al. A meta-analysis of the medium- and long-term effects of laparoscopic sleeve gastrectomy and laparoscopic Roux-en-Y gastric bypass. BMC Surg 2020; 20:30. 